\title{
Retroperitoneal abscess and retropneumoperito- neum arising from a gastric intramural abscess: a case demonstrating the gastric bare area
}

\author{
Soyoung Park and Myung-Won You
}

Department of Radiology, Kyung Hee University Hospital, Seoul, Korea
Received: March 25, 2020

Revised : April 7, 2020

Accepted: April 8, 2020

\section{Correspondence to}

Myung-Won You, M.D.

Tel: +82-2-958-9502

Fax: +82-2-960-8707

E-mail:ruruu77@gmail.com https://orcid.org/0000-00016262-5784
A 74-year-old man presented with a 10day history of nausea and vomiting. $\mathrm{He}$ had been diagnosed as having diabetes mellitus, for which he was followed at a local clinic. Laboratory data revealed hypotension (6o/45 $\mathrm{mmHg}$ ), leukopenia $(2,850 / \mu \mathrm{L})$, and elevated C-reactive protein levels $(38.97 \mathrm{mg} / \mathrm{dL})$, which were indicative of sepsis. Although no acute signs or symptoms were observed, immediate computed tomography (CT) study was performed to determine the cause of the sepsis. The scout image showed a collection of mottled gas surrounding a kidney shadow within the left abdomen, indicating a retropneumoperitoneum (Fig. 1A). CT images showed an intramural rim-enhancing fluid collection at the gastric fundus, presumably an intramural abscess. The gastric wall of the esophagogastric (EG) junction and the fundus were poorly visible, and an extensive retroperitoneal abscess and air collections were found within the left anterior and posterior pararenal spaces (Fig. 1B and ${ }_{1 C)}$. We suspected sepsis due to a gastric retroperitoneal perforation, and the patient was referred for emergent surgery. Necrosis of the gastric fundus and EG junction, and profound retroperitoneal inflammation were found during surgery; therefore, a proximal gastrectomy and delayed esophagojejunostomy were performed. Microscopic examination revealed a transmural

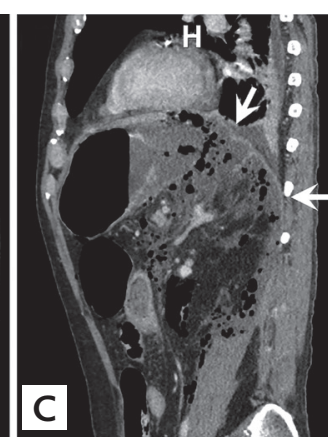

Figure 1. Contrast-enhanced computed tomography images of the abdomen. (A) The scout image shows mottled gas collections surrounding the left kidney (arrows) and extending longitudinally within the left abdomen. (B) Axial and (C) sagittal planes show an intramural abscess of the proximal stomach involving the posterior fundus and esophagogastric junction (B, upper arrow), and an air-containing abscess spreading into the gastric bare area (B, lower arrows; $\mathrm{C}$, arrows) and left anterior and posterior pararenal spaces. 

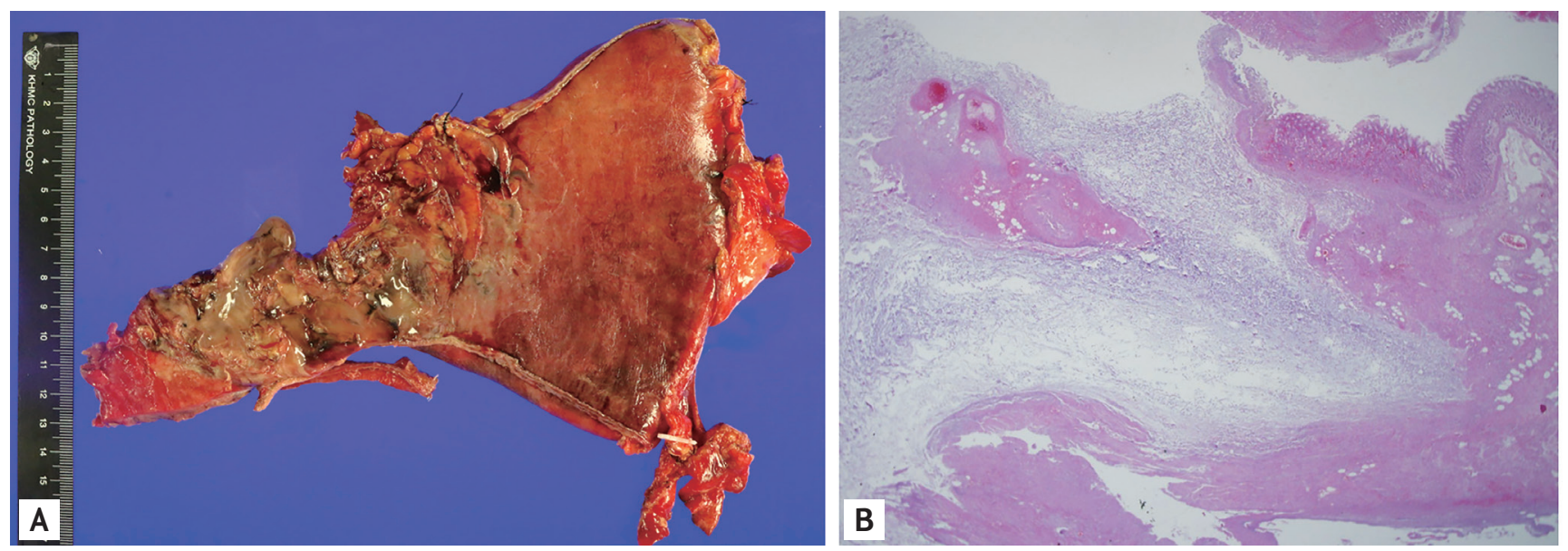

Figure 2. Pathological examination results. (A) Gross specimen of the resected stomach showing necrosis and perforation of the esophagogastric junction and fundus. (B) Microscopic examination result $(\mathrm{H} \& \mathrm{E}, \times 4 \mathrm{O})$ showing a transmural infarction and submucosal abscess of the proximal stomach.

infarction with a submucosal abscess involving the EG junction and fundus (Fig. 2). The patient was doing well at 1-year postoperative follow-up. Written informed consent was obtained.

In this case, the patient showed no acute signs or symptoms that would have indicated a surgical abdomen. Instead, he presented with vague symptoms, including nausea and vomiting. An unusual retroperitoneal perforation of a gastric abscess, manifesting vague signs and symptoms, masked the surgically emergent condition. However, in a patient with diabetes mellitus, sepsis may warrant further evaluation. Retroperitoneal extension of a gastric abscess is an unusual but possible condition because of the extraperitoneal portion of the stomach. This is the gastric bare area, which encroaches upon the posterior surface of the gastric fundus and subcardial portion. Proximal gastric cancers or inflammation can invade the gastric bare area and, from there, the retroperitoneum.

\section{Conflict of interest}

No potential conflict of interest relevant to this article was reported. 